\title{
Redaktion
}

\section{Über die Schwierigkeiten, mit den Problemen des Terrorismus fertig zu werden}

Schon seit Jahren sprechen wir von ,"Tendenzwende“, von der ,,zweiten Restaurationsphase“ (nach dem ersten antikommunistischen, restaurativen ,roll back ${ }^{6}$ unter Adenauer in den 50er Jahren), von der Entwicklung der Bundesrepublik Deutschland zur autoritären Demokratie. Eine Reihe von Gruppen und politischen Theoretikern gar hegt die Auffassung, die westdeutsche Bundesrepublik befinde sich auf dem besten, oder besser gesagt: auf dem schlechtesten Wege in einen ,neuen Faschismus“. Tatsächlich ist nach der durch die Studenten- und Jugendlichenrevolte aufgewühlten, offenen, Entwicklungsphase der 60er Jahre der Normalität deutscher Obrigkeitsstaatlichkeit wieder Geltung verschafft worden. Die eingeleiteten Reformen wurden rückgängig gemacht, gestoppt oder so verbogen, daß außer Effizienz nichts mehr; vor allem kein Körnchen Emanzipation, übrig bleibt. Der Staat der Bundesrepublik Deutschland zeigt, wie Franz Josef Strauß es ausdrückte, seine Zähne. Und daß dieser Staat zuzuschlagen versteht, hat nicht nur die spektakuläre Aktion von Mogadishu weltweit deutlich gemacht. Aber auch in den übrigen Bereichen der Öffentlichkeit, der gesellschaftlichen Institutionen und Organisationen ist die Tendenzwende kennzeichnendes Merkmal der gegenwärtigen Phase der westdeutschen Entwicklung. Den Berufsverboten nach den Minister präsidentenerlassen von 1972 im staatlichen Bereich folgten die Unvereinbarkeitsbeschlüsse in den Gewerkschaften. Im Bildungswesen ist die Lockerheit antiautoritären Verhaltens in Unterricht und Studium vorbei, im Justizapparat wird durchgegriffen. So eine ,schlappe“ Verhaltensweise wie die Amnestie für Demonstrationsstraftaten im Jahre 1969 unter der Kanzlerschaft Willy Brandts wäre gegenwärtig undenkbar. Die ,, heỉßen“ Herbste und Sommer der späten $60 \mathrm{er}$ Jahre sind durch die frostige Kälte einer durch und durch autoritären Entwicklung der gesellschaftlichen Verhältnisse in der Bundesrepublik abgelöst worden. Dabei wirkte das Ende der ökonomischen Prosperität, der Übergang zur Dauerkrise mit Massenarbeitslosigkeit wie ein Verstärker. Austerity, d. h. eine Politik der ökonomischem Restriktionen und der politischen Strenge, wurde - übrigens imganzen von der Krise betroffenen Westeuropa zum Begriff für das gegenwärtige staatliche Handeln. Ökonomische Krise, Rechtsentwicklungen im Parteiensystem der BRD und die niemals in der jüngeren deutschen Geschichte aufgearbeitete obrigkeitsstaaliche preußisch-deutsche Tradition definieren das Terrain, auf dem wir alle uns in unserer politischen, aber nicht nur politischen Existenz bewegen.

\section{Die autoritäre Demokratie ist noch kein neuer Faschismus.}

Der Prozeß einer autoritären Entwicklung war und ist keineswegs geradlinig. Er vollzieht sich in Brüchen und Sprüngen. In dieser Hinsicht war das Jahr 1977 ganz entscheidend, vielleicht eines der für die zukünftige Entwicklung entscheidendsten Jahre nach dem Zweiten Weltkrieg. Denn die Terroranschläge auf Buback, Ponto, Schleyer haben wie Katalysatoren bei der Rückwärtsentwicklung in die autoritäre Demokratie funktioniert. Die Geschwindigkeit, mit der Verteid igerausschlüsse bei politischen Prozessen, die skandalöse Kontaktsperre der politischen Gefangenen durch die Institutionen der parlamentarischen Demokratie rechtmäßig (im rechtsstaatlichen Sinne) gemacht wurden; die Selbstverständlichkeit, mit der polizeiliche Übergriffe, wie gegen die Kernkraftgegener in Grohnde, Brokdorf oder Kalkar in der Öffentlichkeit hingenommen wurden und mit Hilfe des geplanten neuen Polizeigesetzes nicht mehr sanktionierbar sein sollen; die vielen anderen in parlamentarischer Arbeit befindlichen Gesetzesentwürfe zum Komplex ,innerer Sicherheit" (insgesamt über 100); die bedrohlichen Tendenzen staatlicher Organe und von großen Teilen der öffentlichen Meinungsträger, die gesamte Linke (von der linken SPD bis zu den K-Gruppen) als ,Sy mpathisanten des Terrors" sozusagen ,out of bound s" zu stellen, zu marginalisieren und dann wohl auch zu kriminalisieren oder doch - wie mit Peter Brückner geschehen zu suspendieren; diese Entwicklungslinien haben die Physiognomie der bundesrepublikanischen Gesellschaft gründlich geklärt. Eine Physiognomie, deren Züge schon lange erkennbar wurden wie auf einem Photo im Entwicklungsbad, und vor dessen Wirklichkeit wir nun alle erschrecken. 
Ist darauf die Fratze eines ,neuen Faschismus“ zu erkennen? Noch nicht. Die Rechtsstaatlichkeit funktioniert: Nicht nur die demokratische Republik sondern auch der einer deutschen, preußischen Tradition entsprechende Obrigkeitsstaat lassen sich rechtsförmlich organisieren. Allerdings bedeutet diese Rechtsförmlichkeit des Rechtsstaates eine Perversion des historiscli gewachsenen Begriffs des bürgerlichen Rechtsstaats. Denn er implizierte die kalkulierbare, förmlich geregelte Begrenztheit staatlicher Kompetenz gegenüber der Gesellschaft, deren freie Regelung einen möglichst breiten Umfang erhalten und behalten sollte. Die Usurpation der Regelung gesellschaftlicher Beziehungen und Konflikte durch den Staat, d. h. ihre Verstaatlichung, führt vom Inhalt des Rechtsstaat immer weiter fort, ohne dabei unmittelbar seine Form zu zerstören; und so ist es entscheidend, daß bei grundsätzlicher Erhaltung der demokratischen, staatlichen und öffentlichen Institutionen, vom Parlament über die Parteien und die Unabhängigkeit der Gerichte bis hin zu den ,interdemiären Gewalten" wie den Gewerkschaften, diese westdeutsche Demokratie autoritär durchstrukturiert wird und dabei die freien Bereiche gesellschaftlicher Auseinandersetzungen enger werden. Gesellschaftliche politische Aktivitäten, die den Normen der verfestigten westdeutschen ,politischen Kultur" nicht entsprechen, werden dabei verfemt, negativ sanktioniert oder gar kriminalisiert, von den Berufsverboten angefangen bis hin zu den sogenannten Gewaltparagraphen, ohne daß damit die Rechtsförmlichkeit der Rechtsstaatlichkeit verletzt würde. Es ist wieder mehr verboten; und in Deutschland ist traditionell alles verboten, was nicht ausdrücklich erlaubt ist, während in anderen Ländern mit republikarischer Tradition eher alles das erlaubt ist, was nicht ausdrücklich verboten ist. Freiheitsrechte sind in dieser autoritären Demokratie aufgehoben oder zumindest gefährdet. Menschenrechte sind verletzt worden, aber ein faschistisches Regime ist die Bundesrepublik deswegen noch längst nicht. Diese Einschätzung hat natürlich politische Konsequenzen, ebenso wie die Definition dieses Landes als ,Faschismus oder ,neuer Faschismus ${ }^{6}$ zu politischen Konsequenzen mit innerer Logik hinführt. Ein faschistisches Regime kann man nur frontal bekämpfen, aus der Illegalität heraus. Gesellschaftliche Bereiche, in denen durch eine kontinuierliche politische Kleinarbeit Positionen gewonnen werden können, wären durch das faschistische Machtsystem verunmöglicht; eine darauf zielende Strategie wäre grundsätzlich verfehlt. Die Notwendigkeit und die historische Aufgabe des Widerstands gegen ein faschistisches Gewaltregime würde auch den ,Griff zur Waffe" rechtfertigen. Aber die Bundesrepublik ist kein faschistisches Regime und daher ist eine solche Strategie des bewaffneten Kampfes nicht nur unsinnig, politisch verfehlt, sondern ihr geht auch die aus dem Widerstand srecht abgeleitete moralische Rechtfertigung ab. Daher gerade rührt die politische und moralische Misere der RAF und ihrer Nachfolgegruppen.

Auch die innere oder äußere Emigration, unter heutigen Bedingungen also die Flucht in die Landkommune oder das in sich ruhende, gesellschaftliche Kontakte möglichst aussparende Alternativprojekt, oder die Reise in ,das Land, wo die Zitronen blühen" (nenne es sich nun Italien oder Tunix) bietet sich als Konsequenz aus der Definition der bundesrepublikanischen Zustände als ,faschistisch" an. Und nicht wenige versuchen diese Konsequenz zu ziehen, unterstützt von einzelnen ,Nouveaux Philosophes“ wie Glucksmann oder Guattari, die in allen Herrschaftserscheinungen ,,mikrofaschistische" Strukturen sehen: im Staat und seinem Handeln ebenso wie in den Strukturen der Arbeiterorganisationen, der Familie und in unseren Köpfen. Diesem Faschismus kann niemand entrinnen, Ausweglosigkeit ist die Folge. Schritte vorwärts zur Befreiung in gesellschaftlichem, kollektiven Zusammenhang, unter Berücksichtigung der Vermittlung von institutionellen und basisdemokratischen Formen können nicht angegeben, vielmehr nur noch denunziert werden als weitere Schritte in Richtung Gulag. Klar, daß die Herrschaft in diesen hochentwickelten bürgerlichen Gesellschaften wie der BRD sich zu totalisieren tendiert. Aber muß daher auch Ausweglosigkeit entstehen? Gibt es nur den Widerspruch zwischen dieser Gesellschaft insgesamt mit Kapital und Arbeit, deren Klassengegensatz auf einmal gegenüber den Herrschaftsmechanismen nebensächlich, gar unbedeutend geworden ist, und den Randbereichen von Jugendlichen, Outcasts, Freaks und Spontis? Man beschneidet sich selbst der Handlungsmöglichkeit in dieser Gesellschaft zur Veränderung dieser Gesellschaft, auch zur Schaffung befreiterer Verhältnisse zwischen den Menschen, wenn mit der Definition dieser Gesellschaft als einer faschistischen die Widersprüche im Herrschaftszusammenhang in allen Bereichen nicht mehr erkannt und ausgenutzt werden. 


\section{Die deutsche Unfähigkeit, den Terrorismus politisch zu verarbeiten}

Tendenzen zur autoritären Demokratie gibt es auch in anderen Ländern als der westdeutschen Bundesrepublik. Nicht diese sind also das spezifisch deutsche Problem. Die Gefahr geht in diesem Lande davon aus, daß sich über diese Entwicklungsrichtung ein repressiv zustande-gekommener Konsens herstellen könnte. Anders als in Ländern wie Italien oder Spanien oder Großbritannien erweist sich die westdeutsche Gesellschaft als erschreckend unfähig, den Terrorismus politisch zu verarbeiten. Nicht nur, daß eine Ursachenanalyse des Terrorismus in der BRD leich thin und demagogisch in "Sympathie" für terroristisch agierende Gruppen oder doch für ihre Ziele umgemünzt werden kann; und nicht nur, daß die Abwehr des Terrorismus ausschließlich als staatliche Aufgabe usurpiert und nicht als gesellschaftlich zu verarbeitende Erscheinung begriffen wird; die Mittel der Terrorismusbekämpfung seitens des Staates und seiner Organe produzieren gerade gesellschaftliche Hilflosigkeit, Machtlosigkeit und daher auf der einen Seite Nonchalance, ohnmächtige Wut, eine verantwortungslose (weil zur Verantwortung überhaupt nicht aufgerufene) Kopfab-Stimmung, Angste, und auf der anderen Seite verrückten Aktionsimus, der in individuellen Terror münden kann, nicht muß.

Um deutlicher zu machen, worum es geht, sei an die Terrorwellen der vergangenen Jahre in Italien erinnert. Die Bombenanschläge und die politischen Morde Ende der 60er und Anfang der 70er Jahre im Rahmen der ,Strategie der Spannung" waren, wie mittlerweile in einer Reihe von Prozessen aufgeklärt worden ist, ausschließlich von der Rechten organisiert, bei aktiver Mithilfe des Geheimdienstes (unter dem General Miceli) und von Teilen der Armee. Auch höchste Politiker regierender Parteien haben dabei ihre Hände im Spiel gehabt. Die Terrorwelle sollte die sozialen und politischen Spannungen im Lande so verschärfen, daß gegen die Erfolge der Arbeiterbewegung im Verlauf der Klassenkämpfe die Law-and-Order-Partei hätte Zulauf finden und politisch Terrain gewinnen können. Es war daher kein Zufall, daß in die gleiche Entwicklungsphase 1971 der Putschversuch des Fürsten Borghese fält. Es ist ausschließlich der Massenmobilisierung - und nicht der staatlichen Terrorabwehr, die eher Bock als Gärtner war - zu verdanken, daß dieser Spannungsstrategie kein Erfolg beschieden war, ja daß die politische Linke in diesen Auseinandersetzungen gestärkt worden ist und Demokratisierungstendenzen in den Betrieben, (Delegiertenbewegung) im kulturellen Bereich (Scheidungsreferendum etc.), in den öffentlichen Einrichtungen (Wahlsiege und Reformpolitik der linken Parteien, vor allem der KPI) durchgreifen konnten. Die Strategie der Spannung versagte, was allerdings kein Ende terroristischer Anschläge bedeutete. Gerade in jüngster Zeit haben die Attentate auf Richter und Staatsanwälte, die Entführungen von Industriellen, die politischen Attentate und Morde an Andersdenkenden in erschütterndem Ausmaß zugenommen. Aber es ist der politischen Öffentlichkeit bis weit ins bürgerliche Lager hinein klar, daß die Terroranschläge eine der verrücktesten, brutalsten, ausweglosesten Erscheinungsformen gesellschaftlicher Zerrissenheit und Perspektivlosigkeit darstellen und folglich nicht durch die staatliche Aufrüstung gegen die Gesellschaft wirksam verhindert werden können. Die alten republikanischen Traditionen und die Erfahrungen aus der antifaschistschen Befreiung haben eine politische Kultur geformt, die gerade in der Verteidigung der republikanischen Freiheiten gegenüber den Unterminierungsversuchen die politische Hauptaufgabe sieht. Demgegenüber tendiert die öffentliche Meinung in der BRD eher dahin, von einer Stärkung des Staates gegenüber der Gesellschaft, also durch seine militärische Niederschlagung, die Bewältigung des Terrorismus zu erwarten. Die dabei mitzertrampelten, verlorengehenden Freiheiten erscheinen dann nur noch als „Preis für die innere Sicherheit“. Sie waren offensichtlich schon vorher wenig wert ...

\section{Betroffenheit und Bedrohtheit der westdeutschen Linken}

Die westdeutsche Linke - ein schwammiger Begriff, aber daher gerade angemessen - ist von diesen Tendenzen in doppelter Weise betroffen. Einmal spürt sie unmittelbar die Einschränkung des Freiheitsspielraums durch die repressiven Reaktionen des Staates und durch eine sich ihr gegenüber hermetisierende Gesellschaft. Zum anderen ist sie den Aktionen der terroristischen Gruppen ausgeliefert, so daß sie nur noch zu reagieren, oft genug nur noch hilflos die Taten und ihre Konsequenzen zur Kenntnis zu nehmen vermag, ohne die Initiative dabei übernehmen zu können. 
Resolutionen, in der die Terrortaten verurteilt werden, reichen beileibe nicht aus. Das war schon im Winter 1974 so, als mit dem Mord an Kammergerichtspräsidenten Drenkmann eine breite Kampagne für menschliche Haftbedingungen, wie sie nach dem Hungertod von Holger Meins anlief, verunmöglicht wurde. Und als eine Bewegung 1975 gegen das Verfassungsgerichtsurteil zum Paragraphen 218 entstand, wurde ihr mit der Entführung des CDU-Vorsitzenden Peter Lorenz das Heft aus der Hand gerissen. Die Morde des Jahres 1977 schließlich haben viele anstrengende Jahre des Kampfes gegen die politische Repression in ihren Erfolgen geschmälert. Denn die Aufklärungsarbeit gegen Berufsverbote und Gewaltparagraphen, gegen die Herstellung des repressiven Konsenses in dieser Gesellschaft darüber, ist dadurch gefährdet, daß nun die Regierenden mit der allgemein als notwendig erkannten Terrorabwehr einen Erdrutsch repressiver Gesetze auslösen können. Zur Betroffenheit der Linken kommt also eine sehr reale Bedrohtheit ihrer politischen und manchmal sogar ihrer persönlichen Existenz.

Davon waren und sind denn auch die vielen Publikationen und Resolutionen zur Terrorismusfrage gekennzeichnet. Angst, Larmoyanz, Trotz, haben in vielen Fällen ihre Aussagen bestimmt. Die eigentlich niemals in der Arbeiterbewcgung und in der politischen Linken strittige Ablehnung der Terrorstrategie wurde und wird (wem gegenüber eigentlich?) nochmals versichert. Nicht, daß dies alles unwichtig, überflüssig gar gewesen wäre, aber mehrere Mängel in den Diskussionen, Publikationen, Resolutionen zu den Terroranschlägen müssen (aus einem, wenn auch noch geringen Abstand heraus und keineswegs mit besserwisserischer Gebärde oder dem Anspruch größerer politischer Radikaiität) konstatiert werden:

1. Über die analy tische Bewältigung der Gewaltphänomene obsiegte häufig die moralische Verurteilung. Damit läßt sich jedoch keines ausgewiesene Position bestimmen, von der aus man politisch handeln könnte. Zu erreichen ist damit allenfails eine gewisse Exkulpation vor denjenigen, die die Terrortaten der westdeutschen Linken anlasten wollen. Das Desiderat, die gesellschaftliche Verantwortlichkeit für die Entstehung des Terrorismus in hochind ustrialisierten Gesellschaften herauszuarbeiten und daher die Strategie gegen den politischen Terrorismus mit einer gesellschaftlichen Veränderungsstrategie zu verknüpfen, ist of genug unter dem moralischen und politischen Durck, insbesondere während der Wochen der Schleyer-Entführung, unberücksichtigt geblieben.

2. Aber auch die bloß analytische Aussage von dem Verhaftetsein des Terrorismus in den Klassenstrukturen der bürgerlichen Gesellschaft bleibt mangelhaft; denn man kann sich erst aus der Initiativlosigkeit lösen, wenn auch moralisch ausgewiesene Zielvorstellungen entwickelt werden. Diese Aussage steht nur scheinbar im Widerspruch zum ersten Punkt. Denn natülich kann es nicht darum gehen, aus einer allgemeinen gattungsgeschichtlichen Ethik die politische Moral abzuleiten. Vielmehr bleiben auch die Moral und die damit verknüpften handlungsleitenden Ideen, Zielvorstellungen, an die Gesellschaft und ihre möglichen, d. h. über den Kapitalismus hinausweisenden Entwicklungsrichtung, gebunden. Die Schwäche der westdeutschen Linken reproduziert sich in einem unproduktiven Zirkel, wenn ihre Handlungsschwierigkeiten aufgrund ihrer Schwäche zur Ursache für Selbstbezogenheit und für die Ablehnung der Anstrengung, politiche Zielvorstellungen zu entwickeln, wird. Manchmal gar führt dies zu einer die gesellschaftlichen Ausgrenzungsmechanismen verstärkenden Selbstmarginalisierung, die überhaupt keine Ausarbeitung von Zielvorstellungen für politische Interventionen in dieser Gesellschaft mehr zuläßt. Es geht ja nicht nur um negative Abgrenzungen zur bürgerlichen Gesellschaft, ihrer Moral und Kultur, und dann um die Entwicklung von alternativen Verhaltensweisen und Verkehrsformen, sondern um zumindest ansatzweise positive Formulierungen dessen, was wir eigentlich wollen.

3. Die Betroffenheit und Bedrohung durch die Terrortaten und die staatlichen Reaktionsweisen darauf haben eine zeitweise Fixierung auf diese Frage bewirkt. Keineswegs abzuleugnen ist deren außergewöhnliche Bedeutung und daher die Notwendigkeit, zu den immer wieder aufgeworfenen Fragen der Gewalt in dieser Gesellschaft Stellung zu beziehen. Aber ist es nicht merkwürdig und bezeichnend zugleich, daß der Flut von Positionspapieren und Resolutionen zur Frage der Gewalt, und zu den Terroranschlägen des letzten Jahres, nur ein schmales Rinnsal von ebensolchen Papieren zum gesellschaftlichen Problem der Arbeitslosigkeit gegenübersteht? Die (sicherlich nicht bewußt manipulierte) Ablenkung von gesellschaftlichen Strukturproblemen durch den Terrorismus und die politischen Konterstrategien haben auch die westdeutsche Linke 
erfaßt. Die Zeitungen (und die Köpfe der Menschen) waren voll von den Anschlägen und von der staatlich organisierten Jagd mit BKA und GSG 9; doch zweifelhaft ist es, ob die tiefergreifenden gesellschaftlichen und individuellen Probleme von dem Rentendesaster bis zur Arbeitslosigkeit deshalb für uns keine so große Bedeutung mehr haben müssen, weil in der Öffentlichketi der „,Kampf gegen den Terrorismus“ Platz 1 eingenommen hat. Wie schon angedeutet, besteht nach unserer Auffassung eine der Hauptaufgaben der Linken darin, die Bewältigung des Terrorismus nicht von den übrigen gesellschaftlichen Widersprüchen und Problemen isolieren zu lassen, sondern gerade den Zusammenhang analytisch und politisch strategisch herzustellen.

\section{Die Verdrängung gesellschaftlicher Zerrissenheit durch den Terrorismus}

Der arbeitslose Familienvater aus dem Bayrischen Wald, der sich in seiner Verzweiflung das Leben nimmt, die 32jährige Lehrerin ohne Anstellung aus Berlin, die von einem Hochhaus springt, oder der ausgeflippte Jugendliche aus Seesen ohne Lehrstelle, der ein Moped klaut, damit abhaut und dabei von einem Polizisten erschossen wird, sind auch Leichen, die den Weg der westdeutschen Entwicklung pflastern. Drücken sie ein weniger ernstes Problem aus als die Toten der Terroranschläge der RAF-Gangster? Man kann nicht Tote gegeneinander aufrechnen und jeder gewaltsam umgebrachte Mensch, ob er nun Schleyer, Meier oder Baumann heißt, ist ein Toter zuviel. Wir müssen uns davor hüten, mit der Fixierung auf die Terrorismusfrage die anderen für Millionenmassen relevanten Konflikte und Probleme aus unserem Gesichtskreis zu entfernen. Das Frappierende in der westdeutschen Entwicklung der jüngsten Zeit ist ja gerade, daß eine sehr breite Schichten ergreifene Betroffenheit von den Terroranschlägen platzgreift, daß in deren Verurteilung, Ablehnung und Bekämpfung so etwas wie gesellschaftliche Synthesis hergestellt wird - und damit zugleich die anderen Probleme der gesellschaftlichen Zerrissenheit überdeckt werden. In der Ablehnung des Terrorismus ist sich die Nation einig; aber wiekommt es trotzdem, daß jährlich über 100.000 Menschen in Untersuchungshaft geraten, daß Millionen Vorbestrafte unter uns sind, daß sich eine den bürgerlichen Normen von Arbeitsamkeit, Disziplin, Verzicht, Anpassung nicht mehr verpflichtete und darauf orientierte , zweite Gesellschaft " herausbildet von all den unter Bedingungen der Arbeitslosigkeit nicht mehr in die bürgerlichen Normen Integrierbaren? Die Einigkeit der Nation in einem Punkt hergestellt ist somit nur eine scheinbare, nur Fassade.

Dies ist aber gerade ein Ausdruck des ,,repressiven Konsenses“ in der westdeutschen Gesellschaft, daß hinter der Wirklichkeit der Fassade die ganze Erbärmlichkeit, Konflikt trächtigkeitt das Leiden und die Fülle der ungelösten sozialen Probleme verschwinden, sozusagen aus der Wirklichkeit der öffentlichen Wahrnehmung verdrängt werden. Über den oben berichteten polizeilichen Todesschuß auf den Jugendlichen in Seesen konnte man in der überregionalen Presse auf der letzten Seite lesen: Vermischtes aus aller Welt, irgendwie unpolitisch, allgemein menschlich. Der Todesschuß des Polizeibeamten Kurras auf den Studenten Benno Ohnesorg am 2. Juni 1967 hat noch eine Bewegung ausgelöst, hat sich dem kollektiven Gedächtnis der westdeutschen Linken als unvergeßbares Datum eingeprägt. Aber ist der Tod des Lehrlings, der Lehrerin, die Selbstverbrennung des AKW-Gegners in Hamburg weniger politisch als der Tod von Ohnesorg auf der einen Seite, von Schleyer, Ponto, Buback, Drenkmann auf der anderen? Was nottut, ist sicherlich auch eine Klärung des Politikverständnisses in unserer Gesellschaft, das die politische Relevanz tatsächlich nur an der Fassade absieht. Farbeier, die nun wirklich die Fassade beschmutzen, werden zur Kenntnis genommen, aufgeregt und empört. Die Gründe, warum Farbeier geworfen werden, die politischen Absichten, die damit bezweckt werden, finden in der Regel keine Beachtung. Es findet so in beinahe jeder Hinsicht ein Verdrängungsprozeß gesellschaftlicher Ursachen und Bedingtheiten statt. Und die Terrortaten werden dann auch nicht mehr in ihrer gesellschaftlichen Bedingtheit gesehen und politisch verarbeitet. Im Gegenteil, sie finden eine Öffentlichkeit, die gesellschaftliche Probleme von mindestens gleicher Relevanz niemals erhalten.

Als gesellschaftliches Problem ist die Terrorismusfrage schon im Ansatz verschoben gestellt, wenn die Neigung zu terroristischen Verbrechen einer bestimmten psychologischen Struktur angelastet wird. Da wurde breit die Rolle der Frau in der Terrorszene reflektiert, da sind dann auch Psychogramme konstruiert, Abnormalität festgestellt oder die Ausbildung an bestimmten Universitäten verantwortlich gemacht worden usw. Aber dies kann ja gar nicht der Kern des Pro- 
blems sein. Man fragt nach der Psychologie der Susanne Albrecht, aber wer fragt schon nach der Psychologie des Franz Josef Strauß? Obwohl doch die seinige politisch für die BRD-Entwicklung sehr viel relevanter sein dürfte als die ihrige. Es wird so das innerhalb eines bestimmten historisch gewachsenen gesellschaftlichen Rahmens ,deviant behaviour", das ja in brutalster Form die Terrorkommandos ausüben, als psychologisches, also als ihr Problem bestimmt, und nicht mehr als ein gesellschaftliches, also auch unser Problem. Die Kopf-Ab-Haltung resultiert genau aus dieser Interpretationsstruktur: Die Probleme des Terrorismus lassen sich lösen, wenn man die Terroristen physisch beseitig. Der Terrorismus ist die Zuspitzung der destruktiven Tendenzen in dieser Gesellschaft, und diese realisieren sich auch in ganz legaler Weise in der Art und Weise, wie die Terroristen beseitigt werden.

Die Verdrängung der gesellschaftlichen Dimension von Gewalt und Terrorismus, die Psychologisierungen und einfachen Zurechnungen sind also verhängnisvoll. Sie entsprechen voll den Ausklammerungsstrategien der bürgerlichen öffentlichkeit, die als Marktöffentlichkeit sich um die Produktion, um gesellschaftliche Ereignisse sowieso nur kümmert, sofern sie sich zu Markte tragen, vermarkten lassen. Die Terrorakte erscheinen ihr als Sensationen, die im Begleitprogramm mit staatstragendem verantwortlichen Bewußtsein zur Schau gestellt werden. Aber der Gesamtbereich gesellschaftlicher Probleme bleibt dabei letztendlich verborgen, oder kommt nur zutage, wenn er ebenfalls sensationell wird.

Für die Linke kann es nicht darum gehen, innerhalb dieser vorstrukturierten Öffentlichkeit auch ihre Position zu den Sensationen zur Schau zu stellen, durch Resolutionen und Publikationen. Fatal ist dies deshalb, weil die Gefahr besteht, an den relevanten gesellschaftlichen Problemen vorbei pohtische Entwürfe zu entfalten und dabei die eigene Schwäche eigentlich zu reproduzieren. Sicherlich ist es notwendig, zu Strategien, wie sie die RAF verfolgt, eine glasklare Position zu entwickeln, die nur in harter Ablehnung des individuellen Terrors bestehen kann, ohne ,,klammheimliche" Freude oder kopf- und identitätslose Abgrenzungen von Positionen aus, die nicht die unseren sind, die uns vorgegeben wurden. Politische Identität in dieser Frage zu finden, scheint uns nur möglich zu sein, wenn politische Strategien entwickelt werden, die die Fülle der gesellschaftlichen Konflikte aufgreifen, dazu Positionen entfalten und Handlungsalternativen aufzeigen. Es darf also nicht immer wieder von neuem passieren, daß durch Terrorakte lähmende Wirk ungen ausgehen. Diese Lähmung ist natürlich nicht nur subjektiver Unfähigkeit geschuldet, sondern ist Ausdruck der realen Situation, in der sich die Linke in der Bundesrepublik befindet. Aber gerade deshalb kommt es wesentlich darauf an, Konzeptionen zu entwickeln, mit denen sich diese Situation verändern läßt und nicht nur in oft genug hilfloser Reaktion auf die Terrortaten kleiner Gruppen, die lediglich der Rechten in die Hände arbeiten, zu antworten.

\section{Politische Fragestellungen, für die wir Antworten finden müssen}

Es erscheint uns angesichts der Vielzahl von Aufsätzen und Broschüren, die in jüngster Zeit zur Frage der Gewalt und Terrorismus herausgekommen sind (insbesondere: „links" Sonderdruck, „Ästhetik und Kommunikation“, „Kritik“, „,Neuer Langer Marsch“, ,SHL-Info"), nicht notwendig, noch einmal die Probleme, die uns die Gewalt der bürgerlichen Gesellschaft und der Terror von kleinen Gruppen aufgeben im einzelnen und daher wiederholend zu behandeln. Daher wird dieses vorliegende Heft auch keinen besonderen Diskussionsschwerpunkt zum politischen Terrorismus in der Bundesrepublik enthalten - entgegen der Ankündigung im Editorial von Prokla 29 -, zumal die dafür vorgesehenen Artikel von Altvater, Blanke u.a. und von Dingel aus zeitlichen Gründen bereits in "Ästhetik und Kommunikation" und der ,Kritik" erschienen sind. Aber die Fragen, die der politische Terrorismus der sozialistischen Linken aufgibt, und erst recht die gesellschaftlichen Probleme, die durch die Sensationen terroristischer Gewalttaten verdrängt werden, werden uns in Zukunft weiter beschäftigen und auch die Veröffentlichungsstrategie der Prokla bestimmen.

Die bisher zur Frage von Gewalt und Terrorismus erschienenen Stellungnahmen, Diskussionsbeiträge, Broschüren und Aufsätze - auch der hier im folgenden von $O$. Kallscheuer abgedruckte - tragen alle mehr oder weniger den durch die aktuellen Ereignisse geprägten Charakter der Überwindung der Sprachlosigkeit, der Artikulation von politischen Standpunkten und der ansatzweisen Analyse verschiedener Aspekte der Bedingungen des Terrorismus. Soll politische 
Diskussion sich nicht nur von Tagesereignissen abhängig machen, sondern sich an den längerfristigen Bedingungen und Praxisformen sozialistischer Politik orientieren, so bedarf es eines weiteren notwendigen Schritts: der intensiven Auseinandersetzung der vorgetragenen Positionen unter der Fragestellung der Handlungsmöglichkeiten und bedingungen der sozialistischen Linken. Folgende Probleme erscheinen uns für eine solche politische Diskussion zentral:

- Welche spezifischen gesellschaftlichen Ursachen führen in der BRD zur gegenwärtigen Form der Terroristenbekämpfung verbunden mit der Stigmatisierung der Linken bis in Gewerkschaften und SPD hinein? Was sind die klassenpolitischen Grundlagen der Entwicklung zur ,autoritären Demokratie" in der BRD? Welche staatspolitischen, rechtlichen und ideologischen Elemente definieren diese ,autoritäre Demokratie“? In welchem Zusammenhang stehen sie zu den spezifisch deutschen Traditionen des preußischen Obrigkeitsstaats, des unbewältigten Faschismus, der fehlenden bürgerlichen Revolution? Welche Rolle spielt dabei die gegenwärtige ökonmische Krise mit den damit verbundenen sozialen Folgen insbesondere der Massenarbeitslosigkeit?

- Warum entsteht der politische Terrorismus in der BRD im Zusammenhang der politischen Linken? Welche politische Kontinuität und Diskontinuität besteht zwischen der APO, der zerfallenden Studentenbewegung und der RAF? Hat sich die soziale Basis der RAF im Kontext der Arbeitslosigkeit von Jugendlichen und Akademikern verändert?

- Besteht ein Zusammenhang zwischen den spezifischen klassenpolitischen Bedingungen der BRD, die sich in der, ,autoritären Demokratie“, der staatlichen Integration des Klassenkonflikts und auch des spezifischen Staatssozialismus der deutschen Arbeiterorganisationen ausdrïkken, und der spezifischen Isolation gesellschaftlichen und politischen Widerstands, sowohl der Linken in Gewerkschaften und SPD, der APO, der sozialistischen Linken, einer Isolation, die sich etwa auch in der RAF in extremer Weise artikuliert?

- Im Vergleich zur BRD: In welchen Formen reagiert der Staat in anderen Ländern - wie hier am Beispiel Italiens angedeutet - auf den Terrorismus, welche gesellschaftlichen und politischen Ursachen hat der Terrorismus dort, und warum reagiert die staatliche Politik nicht in Form einer autoritären Demokratie?

- Wenn die politische Isolation einer Opposition in der BRD die Grundlage sowohl der RAF, des Zerfalls der Studentenbewegung, aber auch die Bedingung einer sozialistischen Praxis ist, welche alternativen Perspektiven hat die sozialistische Linke? Welche alternativen Formen von Arbeit und Bedürfnisbefriedigung und damit Widerspruchspotentiale entstehen im Zuge der gesellschaftlichen und politischen Entwicklung der BRD, und in welcher Form sind sie politisierbar? Wie verhält sich die sozialistische Linke zur SPD und Gewerkschaften? Wie verhält sie sich zur bürgerlichen Demokratie? Welche Ziele kann die sozialistische Bewegung formulieren, welche Kriterien für gesellschaftliche Veränderungen hat sie entwickelt und muß sie entwickeln, welche Formen der Organisation zur Verwirklichung der Ziele sind geeignet oder ungeeignet?

Dies sind nur einige Fragen, die sich im Zusammenhang einer Terrorismusdiskussion aufdrängen und die eine Diskussion um die Bedingungen und Praxisformen sozialistischer Politik in der BRD beatnworten muß. Die Prokla wird versuchen, hierzu Analysen anzuregen und zu veröffentlichen. 
ken Diskussion bisher nahezu völlig vernachlässigt, was meiner Meinung nach Ausdruck davon ist, daß sich die sozialistische Linke die Frage nach ihrer eigenen politischen Kultur noch nicht gestellt hat. Um einige der Schwierigkeiten dabei, diese Frage zu stellen (geschweige denn, sie zu beantworten) soll es im folgenden gehen.

\section{II.}

\section{Doch zuvor noch zwei Randbemerkungen:}

Erstens glaube ich nicht, daß Senator Glotz in seinen politischen Äußserungen gegenüber den Herausgebern des ,Buback-Nachrufs“ seiner Diagnose von den ,zwei Kulturen“" treu geblieben ist, wenn nämlich seine - in anderen Punkten sicherlich diskussionswürdige (11) - Kritik auf die Forderung nach einer Art Vertrauensvorschuß für das Staatswesen der Bundesrepublik hinausläuft. Wer (z.B. durch eine Dokumentation, die dem Denken und Empfinden der ,zweiten Kultur" in der öffentlichen Diskussion einen Platz einräumen soll) nicht von vorneherein dem öffentlichen Konsens zuzuordnen ist, dem könne gar vorgeworfen werden, er trage zur ,Zerstörung der politischen Kultur" der Bundesrepublik bei.

Solche Argumentation zeichnet sich wohl kaum durch Logik aus. Denn: dem Auseinanderfallen ,zweier ganz verschiedener Kommunikationssyteme“ ist sicherlich nicht dadurch beizukommen, daß eines der beiden zum einzig legitimen erklärt und damit das andere ausgegrenzt wird (um dann - wohl nicht von Senator Glotz, aber, um mit unseren Hypothesen im Bereich des Möglichen zu bleiben, vielleicht von einem christdemokratischen Nachfolger - später kriminalisiert zu werden ... .). Zeugt solche Ausgrenzung von ,,politischer Kultur"? Oder vielmehr: von welcher?

Zweitens wäre nämlich auch zur offiziellen politischen Kultur der Bundesrepublik Einiges mehr zu sagen. Es muß allerdings sehr in Zweifel gezogen werden, ob die hiesige obrigkeitsstaatliche politische Kultur - in ihren beiden Varianten von , akklamatorischem " und ,legalistischem ${ }^{6}$ Staatsverständnis (12) - in der Nachkriegsgeschichte der Bundesrepublik überhaupt jemals zum Abbau von Kommunikationsschranken in der Gesellschaft beigetragen hat. Damit ist $u$. a. das Fehlen einer echten, nationalen republikanischen Tradition angesprochen (13), das die deutsche Nation z. B. von der französischen oder italienischen unterscheidet und in Westdeutschland eine Auseinandersetzung mit der faschistischen Vergangenheit verhindert hat. Der BRD-Staat bestimmte seine Identität zunehmend weniger durch positive Füllung von Demokratie als durch die Ausgrenzung von ,Feinden der Demokratie‘. So ist in der Tat die westdeutsche politische Kultur nie ohne eine „Freund-Feind-Linie" ausgekommen: Die durch die ,unbewältigte Vergangenheit" hart angeschlagene nationale Identität wurde in den ersten Jahren der deutschen Spaltung negativ gegenüber dem ,äußeren Feind' (dem internationalen Kommunismus, Moskau, der DDR ... ) müh-

11 vgl. Blanke/Christoph Kreuzberger/Müller/Schäfer, „Über politisches Handeln ...", a.a.O., S. $52 \mathrm{ff}$.

12 s. H. Gerstenberger, SHL-Info Nr. 10); vgl. auch die Ausführungen von Blanke u.a. zur sozialdemokratischen Vorstellung einer bereits Demokratie garantierenden ,parlamentarischen Bereichsrationalität'; „In geradezu klassischer Weise versucht die Sozialdemokratie, ihre linken Kritiker abstrakt auf einen parlamentarisch-demokratischen Rahmen zu verpflichten. Zugleich verändert sie durch ihre konkrete Politik diesen Rahmen ständig in Richtung auf einen autoritären politischen Zustand. Diese ,paradoxe' Handlungsweise kann gerade das Gefühl des ständigen Angeschmiertseins und Ausweglosigkeit produzieren, das manche Studenten zum politischen Wahnsinn treibt." (Kritik, Nr. 15, S. 54 f.)

13 vgl. O. Negt, „Die Misere der bürgerlichen Demokratie in Deutschland“, in: ders., ,Keine Demokratie ohne Sozialismus. Über den Zusammenhang von Politik, Geschichte und Moral“", Frankfurt/M. 1976, S. 17 ff 
sam stabilisiert, um dann - wie Jürgen Seifert gezeigt hat (14) - zunehmend auf die Front gegenüber dem, inneren Feind" verschoben zu werden. (Nach Mogadisciu scheint nunmehr in der Kombination von ,internationalem Terrorismus" und den zu seinen hiesigen ,Sympathisanten" deklarierten sozialistischen Kritikern von Staat und Gesellschaft ein für die aktuelle ,sozialpsychologische Kriegsfuhrung' brauchbares neues Feindbild gefunden zu sein, das gleichzeitig ,innerer' wie ,äußerer' Feind ist.)

\section{III.}

Soviel vorläufig zur politischen Kultur der BRD, ohne die auch der Mescalero nicht verstanden werden kann. Was mich an dieser Stelle interssiert, sind jedoch die z. B. im ,Buback-Nachruf" oder anderen Blättern der „Info-Kultur" (wie Senator Glotz sie genannt hat) zum Ausdruck gebrachten Haltungen selbst: Haltungen, Ansichten, Gefühle eines großen Teils der ,linken scene", die gar nicht mehr Aufnahme in die ,offizielle Kultur" finden und die im Zeichen der, Sympathisanten'-Hatz nunmehr offen unterdrückt, ausgegrenzt, illegalisiert werden. (In Berlin hat man sich unter fadenscheinigem Vorwand - sie hätten sich mit Druckerzeugnissen ,identifiziert" die Drucker des Agit-Kollektivs herausgegriffen.)

Warum ist die Auseinandersetzung mit diesen Gefühlen - auch wenn sie wie beim Mescalero als „Rülpser“ auftreten - notwendig und sinnvoll? (Eine Auseinandersetzung, von der viel geredet, die aber kaum praktiziert wird). Eine - durch die aktuelle Entwicklung von Staat und Gesellschaft der BRD beförderte - völlige Trennung der ,,beiden Kulturen“, der Info-Kultur und der offiziellen Kultur, ein völliger Abbruch der Kommunikation, wie er durch Zensur-Paragraphen und willkürliche Polizeieinsätze und Verhaftungen vorangetrieben wird, ist die beste Methode, echte Sympathisanten mit dem Terrorismus hervorzubringen. Gewalt - gegen Menschen ist eine mögliche Konsequenz der Unfähigkeit zur Kommunikation.

\section{IV.}

Daß es sich bei solchen Befürchtungen nicht um bloße Spekulationen handelt, läßt sich an der aktuellen Entwicklung in Italien feststellen. Dort sind es nicht mehr nur „Zwei Kulturen“, von denen für die BRD der Sozialdemokrat Peter Glotz spricht, sondern - wie der KPI-Theoretiker Alberto Asor Rosa schrieb - „zwei Gesellschaften": Die erste, offizielle Gesellschaft umfaßt u. a. auch die organisierte Arbeiterbewegung, die beschäftigten Lohnabhängigen, kurz alle diejenigen, die innerhalb des gesellschaftlichen und politischen Systems Ausdrucks-, Handlungs- (und Veränderungs-) Möglichkeiten finden; die zweite Gesellschaft umfaßt all die, die dies nicht mehr tun oder können u.a. die Arbeitslosen, prekär oder schwarz Beschäftigten (v.a. Frauen) und große Teile der Jugendlichen, der Studenten oder Akademiker ohne

14 s. Jürgen Seifert, ,Innerer Feind und Restauration. Seine Bestimmung und Behandlung in der Geschichte der BRD“", in: Links, Jan. 77 (Sondernummer über politische Unterdrükkung und Repression in der BRD), S. 25 ff. 
Aussicht auf sinnvolle Beschäftigung.

„Der politisch entscheidende Punkt" - so Asor Rosa an die Adresse seiner KPIGenossen - ,liegt in folgendem: Wir müssen uns fragen, was wir für diese zweite Gesellschaft getan haben, die neben der ersten gewachsen ist, oder vielleicht eher auf Kosten der ersten, jedoch ohne daraus relevante Vorteile zu ziehen, ohne einen Ausweg zu haben und ohne eine wirkliche Verankerung in der, ersten Gesellschaft ${ }^{\star}$. Ich will - als notwendige Präzisierung - noch hinzufügen: Wir haben die Wahl getroffen, die ich für richtig hatle, einen Typ von Transformationsgesellschaft zu verteidigen, in deren Zentrum - soweit es uns als Partei betrifft - die organisierte Arbeiterklasse steht. Heute besteht die Gefahr darin, daß alles, was nicht in diesen Gesellschaftstyp eingeht - also ,emarginazione' (15), Arbeitslosigkeit, Jugendarbeitsiosigkeit, ,disgregazione" $(15)$ - sich gegen ihn wie ein zerstörerischer Wirbelwind entlädt." (16)

Auch hier erscheint nur die erste, institutionell organisierte Gesellschaft, v.a. die organisierte Arbeiterbewegung als Träger positiver Werte der Gesellschaftsveränderung: Diese Trennung, die in Italien zu recht als zynisch empfunden und kritisiert worden ist (17), wurde noch vertieft durch die von der KPI versuchte Entgegensetzung von ,zwei Moralitäten": der Arbeitsmoral der organisierten Arbeiterbewegung, die Strenge und Sparsamkeit (,austeritå), Effizienz und Staatsbewußtsein impliziert - und den aktuellen Aufgaben der Kommunisten als ,Regierungspartei“ entspricht und der Bedürfnismoral (,teoria dei bisogni') der arbeitsunwilligen Studenten, der Ausgeflippten der Warenhausgesellschaft usw....

Die Explosion der ,neuen italienischen Studentenbewegung' im Februar 77, auch die dadaistischen Aktionsformen der ,Stadtindianer' (der Vorbilder des Göttinger Mescalero) waren Versuche eines Teils dieser „Zweiten Gesellschaft“, der ohne jede staatliche Unterstützung am Rande der Universität lebenden ,nicht-garantierten " Studenten, sich zur Geltung zu bringen - Versuche, die gescheitert sind. Das Theatralische der ,indianischen "Aktionen, ihre spielerische Ironie waren - was ihre ausländischen Fans in Berlin, Frankfurt oder Paris übersehen oder verdrängt haben -

15 ,emarginazione ${ }^{c}=$ Verdrängung (vom Arbeitsmark $t$ ) und ,disgregazione ${ }^{c}=$ Zersetzung beziehen sich im italienischen politischen Sprachgebrauch nicht allein auf die Herausbildung eines ,sekundären Sektors' auf dem Arbeitsmarkt (in dem, je nach Region, die Übergänge zwischen Heimarbeit, ,marginaler Beschäftigung", Schwarzarbeit und völliger Beschäftigungslosigkeit fließend sind), sondern auf soziale und psychologische Handlungsstrukturen wie ,Randgruppenbewußtsein', der ,Zersetzung'von politischer Handlungsfähigkeit. Der kommunistische Sprachgebrauch sieht hier allerdings nur die negativen Seiten gesellschaftlichen Integrationsverlustes und läßt die positiven Versuche neuer Identitätsfindung - z. B. die kulturrevolutionären' Aspekte der Frauenbewegung, Studentenbewegung etc. - aus der Betrachtung heraus. In dem Maße, wie die KPI allerdings selbst von jener ,Disgregation' angesteckt wird, hat sie seit Herbst 77 eine Kurskorrektur versucht.

16 Dieser Artikel (Unità, 20.2.77) wurde in die gleichnamige Aufsatzsammlung von A. A sorRosa aufgenommen: „Le due società. Ipotesi sulla crisi italiana“, Torino (Einaudi editore) 1977, hier S. 64

17 Vgl. die Beiträge in: aut aut No 161 (sett.ott. 1977) sowie die entsprechende Diskussion in der Tageszeitung il manifesto, s. a. Sergio Bologna, „La tribu delle talpe“, in: Primo Maggio No 8 (1977), S. 3 ff. 
keineswegs Ausdruck von Freude, sondern von (u. a. materieller Existenzunsicherheit, tiefer Traurigkeit (die sich selbst nicht mehr, ernst "nimmt), Perspektiv- und Sinnlosigkeit aller den ,Nicht-Garantierten ' in der Gesellschaft, auf dem Arbeitsmarkt, in den Institutionen offenstehenden Wege. Ihr kreativer ,Nihilismus' hatte durchaus seinen Hintergrund in einer sinnlos werdenden gesellschaftlichen Erfahrung, so daß für triumphalistische Umdeutungen, wonach ,d iese Bewegung, die es erreichen wird, die gigantische kapitalistisch-bürokratische Maschinerie zu zerstören, a fortiori durchaus fähig sein wird, eine andere Welt aufzubauen" (18), kein Anlaß bestand.

Verzweifelte Ironie bestimmte auch zunächst das Verhältnis dieser ,Nicht-Garantierten ${ }^{6}$ zum politischen System, nachdem sich die KPI (die die meisten auch der Stadtindianer am 20. Juni 1976 gewählt hatten!) durch ihre Haltung des, Nicht-Mißtrauens' gegenüber der christdemokratischen Regierung aus einer Oppositionspartei $\mathrm{zu}$ einer Partei der Regierungsmehrheit ohne Regierungsmacht transformiert hatte und damit der ,zweiten Gesellschaft" als Teilhaber des alten Machtsystems erscheinen mußte (19). ,Zwischen dem System und den Kräften der studentischen Protestbewegung" - so wieder in seiner illusionslosen Sprache A. Asor-Rosa, KPI-Mitglied und Universitätsprofessor (,roter Baron') an der römischen Universität - ,gab es nur noch die Gewerkschaft und die KPI (aber in erster Linie die KPI), um die Beweggründe jener ,ersten Gesellschaft" $\mathrm{zu}$ vertreten "; an den völlig desorganisierten italienischen Massenuniversitäten war, die KPI somit ,die einzige Institution, die als .solche überlebt hatte und gleichzeitig gesellschaftlich verankert, sichtbar und faßbar war" (20). Gegen sie also richtete sich die Verzweiflung einer Bewegung, die sich zunehmend als anti-institutionelle, anti-politische verstand. So gesehen bedeutete die Identifikation mit der Indianerrolle auch ein ironisch-resignatives ,Anerkennen der eigenen Situation des Ausgeschlossenseins (21).

Indem aber die Bewegung bisher scheitert (aus Gründen, auf die wir hier nicht ausführlicher eingehen können (22)), in dem Maße, wie sich ihre Kontinuität nur durch Kampf(Re)Aktionen auf von außen gesetzte Anlässe - zumeist Polizeiprovokationen - herstellt, schlägt die gesellschaftliche ,Zersetzung' (s. Anm. 15) auch auf ihr wichtigstes Potential durch: auf ihre alternative Art, Politik als nicht mehr vom eigenen Lebenszusammenhang getrennt zu begreifen, sie als, Wiederaneignung des Lebens' zu versuchen (oder wenigstens zu ,inszenieren'). Dies läßt sich am Kräfteverhältnis zwischen den ,kreativen Autonomisten" und der ,militarisierten Autonomie“ innerhalb der Bewegung ablesen. Läßt sich die ;kreative“ Identitätsfindung der neuen Studentenbewegung mit der Liedzeile aus der italienischen Frauenbewegung ,Wo die Macht aufhört, entsteht die Hoffnung" programmatisch beschreiben, so treibt die

18 Felix Guattari, ,Millionen und Abermillionen potentieller Alices“, Vorwort von: Kollektiv A/traverso, ,,Alice ist der Teufel. Praxis einer subversivern Kommunikation. Radio Alice (Bologna)", Merve-Verlag Berlin 1977, hier S. 14

19 vgl. die Diskussion in Prokla, Nr. 26, 27, 29 sowie Thomas Bieling, „16 Thesen zur italienischen Studentenbewegung" (Januar 78, bisher noch nicht veröffentlicht, erscheint voraussichtlich in Prokla 32)

20 "Le due societa “, a.a. O., S. 66

21 s. Jens Huhn, „Auf dem Kriegspfad - die Stadtindianer“, in: Links, Dez. 77, S. 15 f.

22 vgl. den in Radikal Nr. 24 (Berlin 24.6.77) abgedruckten Bericht Carlo Donolos über die „Studentenbewegung in Italien". 
,organisierte Autonomie innerhalb der Bewegung rücksichtslos Machtpolitik. In dem Maße, wie die „Partei des bewaffneten Kampfes“, die „Genossin (Pistole) P 38“ innerhalb der ,Autonomi“" an Einfluß gewinnt (23), droht die Identität dieser neuen gesellschaftlichen Befreiungsbewegung der ,nicht-garantierten" Studenten kaputt zu gehen: In Rom und Bologna, also dort, wo die ,Autonomi“" in der Studentenschaft einen gewissen Einfluß haben, wird es für die pazifistischen unter den ,Autonomi“", die nur ihre „Betroffenheit" artikulieren, alternative Verhaltensformen, „Politik in erster Person" erproben wollen, für die Flippis oder Alternativ-freaks auf den permanenten Vollversammlungen zunehmend unmöglicher, sich gegen diejenigen durchzusetzen, die aus der Uni heraus militante Demos organisieren, bei denen dann Autos in Brand gehen, Mollis fliegen und auf Polizisten geschossen wird. (All dies muß natürlich auch vor dem Hintergrund der von Neofaschisten und Teilen des repressiven Staatsapparats vorangetriebenen "Spannungsstrategie" gesehen werden).

Diese wenigen Bemerkungen mögen als Einstieg genügen. Auf die neueren Versuche der Selbstkritik der KPI in ihrer Haltung gegenüber der Jugendbewegung, auf die innerhalb der Neuen Linken beginnenden Prozesse des Umdenkens (24), auf die neuen von Gewerkschaften und Linksparteien unterstützten Arbeitslosen-Initiativen soll - da das nicht Thema dieses Beitrags ist - hier nicht eingegangen werden.

V.

Die Entstehung von alternativen „scenes“, von „drop outs" in allen kapitalistischen Metropolen (es hat ja nicht zufällig in den 60er Jahren in den USA angefangen) ist Zeichen des Integrationsverlusts alter Werte von Arbeit, Ordnung, Rationalität; Anzeichen auch eines Überdrusses an (Partei-)Politik. Mit der Gefahr der Übersteigerung kann man doch von ersten Anzeichen einer umfassenden Zivilisationskrise sprechen.

Mit Zivilisationskrise meine ich den beginnenden Zerfall von identitätsstiftenden Momenten der bürgerlichen Gesellschaft (,bürgerlich“ hier im Sinne von ,societả civile (25)). Doch ist diese „Amerikanisierung“ kein Prozeß, dem Sozialisten freudig - oder bestenfalls achselzuckend - gegenüberstehen könnten. Bedeutete er doch wenn er wirklich durchgreift - auch den Verlust von Kommunikationsfähigkeit in der Gesellschaft, ihre Zersetzung in ein Mosaik von Ghettos, ,scenes“ oder „Stämmen"; und damit auch eine weitere Entkoppelung von politischer Herrschaft und Legitimation.

(Am Ende eines solchen Zersetzungsprozesses stünde somit eine Art neuer autoritärer [z.B. Polizei- oder Atom-] Staat, dem nur noch einige „communities“ am Rande der Gesellschaft Widerstand leisteten; ,Sozialismus' stünde nicht mehr alter-

23 vgl. den Brief über „Au tonomisten, Stadtindianer, Bewaffnung“" in Autonomie Nr. 8/Aug. 77 (Trikont-Verlag), S. 74 f.

24 Silviero Corvisieri, „Die Notwend igkeit einer Wende“, il manifesto, 3.9.77

25 vgl. Jürgen Habermas, „Können komplexe Gesellschaften eine vernünftige Identität ausbilden?", in: ders., ,Zur Rekonstruktion des Historischen Materialismus“, Frankfurt/M. 1976, S. 92 ff. sowie die Einleitung zu diesem Buch, v.a. S. $30 \mathrm{ff}$. 
nativ zu ,Barbarei ${ }^{6}$, sondern entstïnde in ihr, an ihrem Rande, in Gestalt von Ghettos, die ,inmitten der entfaltesten Todeskultur mit Leben experimentieren“"(26), die neuen „Kulturen“" nährten sich - gleichsam biologisch - vom Zersetzen, von der Fäulnis der alten ...))

Natürlich ist ein solcher Prozeß weder bereits gelaufen, ist sein Ausgang bereits absehbar - wir beginnen gerade erst, seine Dimensionen zu ahnen - noch ist er ,objektiv': Er kann nämlich nur dann (alternativlos) stattfinden, wenn die gesellschaftlichen Subjekte tatsächlich auf kommunikatives Handeln verzichten, wenn sie die Bereitschaft zu rationaler Diskussion und Handlungsorientierung mit zu den leeren Coca-Cola-Büchsen auf den Abfallhaufen schmeißen. Und hier beginnt auch das Problem und die Verantwortung einer ,politischen Kultur“ von Sozialisten. Diese kann (wenn die gerade skizzierte Apokalypse eine vielleicht pessimistisch übersteigerte, aber doch reale Gefahr zum Ausdruck bringt) keine bloß partikulare, kein bloßes, Wir-Bewußtsein $^{6}$ eines ,linken Lagers' mehr sein, sondern muß immer auch gesamtgesellschaftlich bezogen und legitimierbar sein. Nur dann können auch die Chancen für Alternativen, die im Aufbrechen von Ungleichzeitigkeiten, im „Patchwork der Minderheiten“ liegen, fruchtbar gemacht werden (27).

Es ist keineswegs paradox, wenn die Aufgabe einer gesamtgesellschaftlich bezogenen „politischen Kultur“ von Sozialisten (v.a. von bundesdeutschen Marxisten (28)) zunächst einmal verlangt, gesellschaftliche Dynamik im Plural zu denken. Denn die oben als beginnende Zivilisationskrise bezeichnete Entwicklung hat sicherlich weitergehende Dimensionen und Ursachen als in der nur ökonomischen Krise des biherigen Akkumulationstypus der kapitalistischen Hauptländer. Ganze Dimensionen (der Krise) des gesellschaftlichen Lebens wie die Frauenfrage oder die politische Ökologie müssen von Sozialisten erst einmal ,entdeckt" und ernstgenommen werden - auch und gerade dann, wenn man die „richtige“ Antwort noch nicht weiß! Das Terrain gesellschaftlicher Auseinandersetzungen - und das läßt sich auch für die in mancher Hinsicht spezifische BRD-Situation feststellen - hat sich im Spätkapitalismus nicht einfach homogen erweitert, sondern vervielfältigt. Und damit entstehen ebenso viele „Kulturen“ oder „Subkulturen“", die je eine spezifische „Betroffenheit" zum Thema haben und sich insofern (erst einmal) ,autonom “ entfalten müssen (29). Die richtige Antwort für die "Totalität" aller gesellschaftlichen Probleme zeichnet sich erst recht bei uns noch lange nicht ab (wenn es sie - im Singular - überhaupt gibt).

26 Editorial „USA“ der Nr. 7 von Autonomie (Juni 1977)

27 Die entgegengesetzte Perspektive (,Die Dekadenz der Wahrheit verschärfen!“") vertritt Jean-François Lyotard in seinem (lesenswerten) Buch ,Das Patchwork der Minderheiten“, Merve-Verlag-Berlin 1977

28 vgl. B. Blanke, ,Kritik und Selbstkritik“, Prokla Nr. 23

29 auch bzw. gerade dann, wenn sie „Arbeitsfelder“ für Sozialisten sind! 
Die kapitalistische Krise mit ihrer Dauerarbeitslosigkeit v.a. für die Jugend und die Gefahren einer ,autoritären Demokratie“ haben aber Auswirkungen auf alle diese Bewegungen und die verschiedenen alternativen ,scenes". Und eine dieser Wirkungen läßt sich in der oben angesprochenen Gefahr der Entstehung einer bloßen GegenKultur, Gegen-Gesellschaft sehen, die alle Kontakte zur offiziellen Gesellschaft abbricht und sich damit selbst ihrer Einwirkungsmöglichkeiten auf diese begibt. Einer solchen Gefahr kann natürlich nicht mit bloßen moralischen oder politischen Appellen begegnet werden. Die Entwicklung einer Akademiker- und Jugendarbeitslosigkeit und der Ausbau des polizeilichen Repressionsapparats, die ideolgische Formierung eines „Totalitarismus der Mitte“, einer „Volksgemeinschaft gegen die Sympathisanten" befördern diese Gefahr unendlich mehr, als Argumente gegen sie ausrichten könnten.

Die Antwort auf diese Gefahr kann somit nur eine gesellschaftliche sein (30), sie muß gesucht, d.h. erkämpft werden in der Fähigkeit der Gewerkschaftsbewegung, die strukturelle Arbeitslosigkeit überhaupt als Problem anzugehen, in der Fähigkeit demokratischer Bewegungen, dem Abbau von Grundrechten wirksam entgegenzutreten, in der Möglichkeit von Bürger- und anderen (z. B. studentischen) „Initiativen“, Probleme wie die Zerstörung unserer Umwelt effektiv in den politischen Willensbildungsprozeß einzubringen ... Nur hier liegen die Mittel, ein Auseinanderfallen "zweier Gesellschaften" zu verhindern. Dies wohlgemerkt nicht, um ,autonomen“" Bewegungen das Wasser abzugraben (z.B.: „Frauen an die Seite der Arbeiterklasse! “), sondern um ihnen den Raum (und nicht nur „Spiel-“ oder „Freiräume") zu geben, der zur Entwicklung einer demokratischen, d. h. von vielfältigen (31) Subiekten getragenen Gesellschaftsveränderung in sozialistischem Sinn unabdingbar ist.

\section{VII.}

$\mathrm{Ja}$ - und? In der BRD bestehen diese Möglichkeiten oder Fähigkeiten für demokratische und gewerkschaftliche Organisationsformen (noch) nicht oder nur in bescheidensten Ansätzen und die sozialen und politischen Aussichten für die nächsten Jahre sind wahrlich nicht rosig. In dieser Situation nun wächst die Gefahr, daß die skizzierten ,objektiven'Tendenzen zum Abbruch der Kommunikation verschiedener "scenes“" oder (Sub), Kulturen“" mit der Gesamtgesellschaft nun auch noch im subiektiven Verhalten dieser „scenes“ ihre Entsprechung finden. Die Linke - um bei uns selbst zu

30 s. Negt, ,Wider die deutsche Verstaatlichung der Konflikte“, a.a.O.

Pierre Victor (nach dem Mai 68 einer der Wortführer der "Gauche Proletarienne") im Nouvel Observateur, 19.9.77: „Wenn die klassischen Liberalen diese radikale Erfordernis begreifen könnten - denn Pluralismus an den Arbeitsplätzen und Lebensorten und nicht nur an der Spitze der Staatsorganisation - so wäre das schon sehr gut. Der Pluralismus beginnt dort, wo die von der Rede und von der Entscheidung Ausgeschlossenen sich versammeln und das Wort ergreifen." 
bleiben - ist heute eine gesellschaftliche, politische und kulturelle „Randgruppe“. Das Verhängnisvollste, was sie tun kann, wäre, daß sie sich auch noch als solche verhält. In Berlin (aber auch in Frankfurt, München oder anderswo) gibt es eine „linke scene", die bestimmtes Sprachverhalten, (oft kümmerliche oder gescheiterte Versuche anderer als der herrschenden Lebensformen - wie Wohngemeinschaften, Kinderläden usw.), einen linken Blätterwald ebenso einschließt wie die (meist nur noch sogenannt) , ,linken Kneipen“" oder die Verlaufsformen studentischer Vollversammlungen. Diese „linke scene“" ist für viele von uns lebensnotwendig, sie bietet Wärme, Schutz gegenüber einem schon ganz anderen offiziell politischen Klima; sie stellt den Erfahrungsraum zur Erprobung alternativer (oft nur anders kaputter) Lebensund Verkehrsformen z.B. zwischen Männern und Frauen dar, Formen solidarischer Alltagsbewältigung, die manchmal der Forderung nach „Sozialismus im Alltagsleben“ nahe kommen. Die "scene“ kann aber auch zum bloßen Refugium vor der Härte und Unbill des Uni-Alltags, des Jobs werden, das dann oft beim Eintritt in den (z.B. Lehrer-)Beruf nicht mehr weiter hilft: Einmal ohne den schützenden Hinter- oder Untergrund der ,scene“ mit der gesellschaftlichen Wirklichkeit konfrontiert, kippen viele sehr schnell um. Einmal im Berufsleben stehend, bleibt vom jahrelangen Kapitalstudium oft nur noch die (arrogante) Einsicht in die ,Notwendigkeit des falschen Bewußtseins" der anderen, ,Normalen" übrig ohne praktische Konsequenzen für das eigene Verhalten. Die so praktizierte ,scene" macht einen also gleichzeitig stark und schwach: stark nach innen, im linken ,wir" (wo der leiseste sogenannte Opportunismus ,kleinbürgerlichen“ Denkens oder Verhaltens schonungslos als „Abweichung“ bekämpft wird ... ), und schwach nach außen (mit den ,anderen' ,kann man ja sowieso nicht reden"...).

In Berlin hat ein Student auf einer Uni-Vollversammlung diese Situation treffend mit dem Bild der "Stadt in der Stadt" beschrieben: „Wir retten uns in die Regel der Wissenschaft, die Bestätigung durch Papiere und Seminare, durch Bücheranhäufung, träumen von der Flucht (Kaffehaus in Kreuzberg, Grassplantagen in der BRD, Farm in Frankreich, etc., etc.). Viele von uns kennen diese Stadt hauptsächlich als (das Universitätsviertel) Dahlem und einen Wust von linken Kneipen und Wohngemeinschaften. Diese Stadt wie die Uni gehören nicht uns, noch nicht einmal zu uns." (32)

Dies ist natürlich nur allein Folge der Formen linker Theoriebildung, der spezifisch „linken Überforderung“ (33), sondern vor allem und in erster Linie davon, daß die Studentenschaft im Uni-Alltag tatsächlich zum Objekt (und im Hochschulrahmengesetz zu einer in Regel studienzeit abzufertigenden Manövriermasse) gemacht wird ...

32 zitiert nach Konsequer/Forum des UStA, Nr. 1, 25.10.77 (UStA = Unabhängiger Studenten Ausschuß = unabhängige, basisdemokratisch orientierte Organisationsform von Studenten der Freien Universität und die in Westberlin derzeit wohl stärkste hochschulpolitische Strömung)

33 vgl. Burkhard Müller, ,,Die Widersprüche überleben“, in: Fuchs/Müller/Ortmann, ,Universitärer Alltag. Lernen, Lehren, und Leben an der Hochschule“, Focus-Verlag Gießen 1977, S. $127 \mathrm{ff}$. 
Trotzdem, wir müssen uns fragen, ob wir nicht mit all den positiven und unverzichtbaren Elementen herrschaftsärmerer Lebens- und Verkehrsformen auch eine Unfähigkeit, „die Widersprüche zu überleben“ (34) mit ,kultiviert" haben, wie sie Z.B. wiederum mit, indianischer Ironie" (die sich selbst nicht mehr ,ernst" nimmt) im Aufruf zum Treffen in TUNIX zum Ausdruck kommt:

„Uns langt's jetzt hier! ...

Wir hauen alle $a b ! \ldots$

Dann steht hier alles leer und still ...

Wir sind alle weg und sehen, daß wir da waren

und sehen, $d a ß$ wir viele'waren, denen es gestunken hat, die genug hatten. Wir sind alle abgehaue weil wir uns unmächtig fühlten und allein und sehen, daß wir gar nicht allein waren.

Und das wollen wir doch mal sehen

ob wir nicht jetzt schon - oder immer noch? - da sind, ob wir nicht jetzt schon viele sind, die gen haben von allem ... Das wollen wir doch mal sehen, ob wir nicht jetzt schon viele sind, die Wide stände leisten in so vielen Bereichen, mit so viel unterschiedlichen Ansätzen, in so vielfältigen Fo men.

Kommen wir also zusammen, bevor wir alle abhauen, auf diesem Widerstandskongreß - erzahlen wi erfahren wir voneinander, bringen wir mal diese Widerstandsbewegung zum Ausdruck - und hauc wir dann zusammen ab, segeln wir alle zum Strand von TUNIX, der weit weg liegen kann oder vielleicl unter dem Pflaster von diesem Land“"

Ich habe hier nur aktuell einige Zeilen und Sprüche aus dem Flugblatt herausgegriffen. Dies nicht, um den TUNIX-Kongreß zu diffamieren, der zum Zeitpunkt, wo ich dies schreibe, erst in zwei Wochen stattfinden soll und hoffentlich ein fruchtbares Gespräch verschiedenster alternativer (Medien-, Schul- Zeitungs- usw.) Gruppen ermöglichen wird. Auch von Senator Glotzs These von den „zwei Kulturen“ wird dort geredet werden. Doch illustriert dieses Flugblat (das Trauer in Lachen verkleidet) ganz gut eine erste Gefahr von bloßem ,scene"-Verhalten, von bloß nach innen verständlichem Wir-Gefühl. Die vermeintliche Kompromißlosigkeit nach innen wird mit der Verhaltensunfähigkeit nach außen erkauft, mit der Gefahr von Selbstisolation, die auch eine Art ,innerer Emigration" werden kann: „Wir, die wir schon eine Weile auf unseren gepackten Koffern sitzen, schlagen vor, daß alle sich bis zum März 1978 aus diesem Deutschland verpfeifen. Wir flaggen unsere Traumschiffe mit den buntesten Fahnen und segeln in den Süden davon - zum Strand von Tunix " beginnt das Flugblatt.

\section{VIII.}

Aus dieser Unfähigkeit zur Kommunikation nach außen hin - die hier nur als eine, allerdings reale Tendenz von ,scene"-Verhalten angesprochen wird, also nicht als arrogantes Abkanzeln der gesamten scene's verstanden werden soll (35) - folgt dann

a.a.O.

35 Dies haben z. B. Tilman Fichter und Siegward Lönnendoncker in ihrem Beitrag ,Die 
unter den aktuellen Bedingungen der Verhärtung des politischen Systems bei einigen eine (immer weniger spielerische) Illegalität als habitus. Diese zweite weitergehende Gefahr im ,,scene“-Bewußtsein mancher Linker zeigt sich z.B. bei Demos daran, daß viele sich mit (Palästinenser-)Halstüchern vermummen. Kann eine Demonstration von Vermummten für die Freilassung der Agit-Drucker aber von Mitbürgern noch als Eintreten für Pressefreiheit verstanden werden? Wenn solche vermummten Scharen mit Fackeln in der Hand dann noch in Sprechchören ,Sympathisanten grüßen die Passanten" rufen, so muß man kein BZ-Leser sein, um die Ironie im Ausdruck ,Sympathisanten" nicht mehr verstehen zu können, sondern diese Horde für echte Terroristen-Anhänger zu halten. (Diese Gefahren zur Selbstisolation gibt es natürlich nicht nur bei der studentischen Linken. H.-J. Benedict hat sehr gut aufgezeigt, wie bestimmte Mobilisierungsformen von ,Berufsdemonstranten ' die Isolation der .Anti-KKW Bürgerinitiativen im März 1977 z. T. mit hervorgebracht haben: „Die neue Grohnder Tradition (Symbol Blume) wurde von der Brokdorfer (Stichwort Helm) abgelöst.“ (36))

Ein anderes Beispiel ist das „Info Berliner Undogmatischer Gruppen“ selbst, dessenwegen die Agit-Drucker im Knast sitzen. „Dadurch, daß für das Info niemand (presserechlich) verantwortlich ist" - schrieb das „Politische Buch“-Kollektiv in der (Abschieds-)Nr. 179 des Info zur Kritik an dessen Selbstverständnis -, ,wird es der Staatsanwaltschaft geradezu aufgedrängt, sich am ,Umfeld' schadlos zu halten. Es geht nicht, daß das Info illegal oder halblegal erscheint und die in der Légalität (im Falle von Gewerbeträgern wie auf dem Präsentierteller) arbeitenden Genossen dafür eingesperrt oder bedroht werden. Das Info illegalisiert dann tendenziell die Buchläden und Druckereien oder ist zumindest willfähriges Werkzeug."

In der (Abschieds-)Nr. 179 des Info hat sich die Mehrheit der Beiträge noch auf die eine oder andere Weise mit diesem (auch die innerlinke Kommunikation letztlich zerstörenden) illegalen Habitus krtisch auseinandergesetzt und war zu dem Schluß gekommen, daß die alte Konzeption eines halb-legalen ,scene“-Blättchens nicht aufrechtzuerhalten sei. Mit der neuen Nr. 181 des „Infos Berliner unfaßbarer Gruppen/Konspi-Ausgabe" werden diese und andere Einwände (z.B. der Stadtteilgruppe Kreuzberg) einfach vom Tisch gewischt: „Wir spüren kein Mißtrauen, wenn wir uns gegen die Bullen fest zusammenschließen“ heißt es nunmehr: „Wir lernen weiter, was in ein paar Jahren jede BI, jede Knastgruppe, jedes linke Kneipenkollektiv beherrschen muß: ein Stïck in den Untergrund zu gehen, um den aufrechten Gang nicht zu verlernen.“ Und: „Meinungsbeiträge sind an den Berliner Untergrund zu richten. Eine Kneipe, eine Gruppe, ein Genosse - lauter Wege“. Hier wird letztlich zy nisch der völlige Kommunikationsabbruch von Ober- und Untergrund bewußt vorweggenommen und damit die von interessierter Seite vorgebrachte These, aus der Linken, aus dem „Sympathisantensumpf“ führe jeder Weg in den Untergrund,

Klammheimlichen im Ghetto“ getan (indem sie die „,zweite KuItur" in der Sprache und mit den Vorurteilen der ,ersten Kultur" einfach als unpolitisch verurteilten), der in der von den Präsidenten der Technischen und Freien Universitäten Berlin herausgegebenen Broschüre ,Zurück zur Politischen Diskussion“ 25.11.77, (S. 6 f.) erschien.

36 s. Hans-Jürgen Benedict, „,Bürger, Linke und Gewalt“, in: Kursbuch, Nr. 48, S. 137 - 152 
indirekt bestätigt.

\section{IX.}

Und hier wird dann eine dritte, in der Ambivalenz von solch halblegalem Selbstverständnis angelegte, Gefahr psychologisch akut: die Gefahr nämlich, daß eine (auch im Umkreis mancher K-Gruppen geschürte) abstrakte Anti-Staats-Haltung in ein Verständnis gesellschaftlicher Konflikte als konkreter Krieg umschlagen kann. Ein solches Verständnis wurde z. B. deutlich in Versuchen, den Mord an Schleyer gegen die Stammheimer ,Selbstmorde" aufzurechnen: Nach der Schlacht zählen beide Seiten ,ihre' Opfer . . . Eine solche Haltung braucht die Faschisierungsthese, die psychologische Bestätigung durch eine völlig feindliche Umwelt: Alle gesellschaftlichen Konflikte können dann nur noch als verschiedene Varianten derselben , totalen ${ }^{6}$ Repression begriffen werden.

Was auch immer in Stammheim passiert sein mag - die offizielle Version des ,Selbstmordes" ist voller Widersprüche und unglaubwürdig: darum unsere Forderung nach einer internationalen unabhängigen Untersuchungskommission! - wer erlebt hat, wie auf einem teach-in Rechtsanwalt Otto Schily auf seine Warnung, die Linke möge sich davor hüten, in ihrem Urteil über den Tod von Baader, Ensslin und Raspe, von der Gewißheit ihrer Ermordung auszugehen, (also gewissermaßen das Vorgehen der staatlichen Stellen zu imitieren und ,kurzen Prozeß zu machen",/ wie Schily und anderen Genossen Rechtsanwälten (Wesel, Spangenberg) eine Welle der Empörung, fast des Hasses aus dem Auditorium entgegenschlug, wer erlebt hat, daß mehr als zweitausend Linke die Aussage Uwe Wesels ,Wir wissen nicht, was in Stammheim passiert ist " nicht ertragen konnten ... der ist geneigt, sich über die psychologische Funktion der Mordthese in der Linken Gedanken zu machen.

„Man mag an eine Ermordung der drei Häftlinge glauben“ - hat Paul Thibaud in diesem Zusammenhang in der Libération bemerkt (27.10.77) - ,aber sich eine opportune Empörung zu fabrizieren, seine eigenen Gefühle soweit zu kanalisieren, daß sie einen blind machen, das ist die alte stalinistische Technik, die man nun interessanterweise bei vielen Anti-Stalinisten wiederfindet. Auf die gleiche Weise gelang es der KPF im Jahre 1956, dadurch bei vielen ihrer Militanten die ,schmerzliche' Affäre der Niederschlagung des Ungarnaufstands durch sowjetische Panzer in den Hintergrund ihrer Probleme zu verdrängen, daß sie auf die antikommunistischen Kundgebungen in Frankreich verwies. Man will also in schwierigen Momente auf jeden Fall verhindern, daß die Geister sich verwirren: man muß sie dann daran erinnern, daß es nur einen Feind gibt, man muß dem Feind wieder Gestalt (des Einzigen, Absoluten) verleihen, was man 1956 tat, indem man den Installationsarbeiter vorzeigte, der bei der Verteidigung des Gebäudes der Humanité gegen unterschiedslos als Faschisten klassifizierte Angreifer zu Tode kam, und was man heute wieder tut."

Die Verengung aller - auch der eigenen - gesellschaftlichen Widersprüche auf die Repression, die Identifikation von ,Stammheim" mit dem ,Modell Deutschland“, die Rede vom ,Faschismus' (statt vom fortschreitenden Abbau verfassungsmäßiger 
Rechte) usw. . . haben also ihre Ursachen weniger in einer theoretisch unklaren Faschismusbegriffs als in einer verlängnisvollen psychologischen Funktion, die - wie der Göttinger Mescalero deutlich genug in seinem ,Buback-Nachruf' beschrieben und kritisiert hat - zu einer ,Identifikation mit dem Aggressor' führen kann: Zu einem Bild von Gesellschaft, die nur noch aus ,Bullen' besteht. Hier wird dann die Abschottung der eigenen Wahrnehmung gegenüber der gesellschaftlichen Realität ,total ${ }^{\star}$ Gewaltphantasien, Klassen- oder „Volkshaß“(!) nicht nur gegenüber „dem System“, sondern gegenüber einzelnen seiner Repräsentanten werden wieder legitim, moralische Barrieren, die oft am Anfang des politischen Engagements standen, können wieder abgebaut werden ... Mit diesen Gewaltphantasien setzte sich der Göttinger Mescalero kritisch auseinander, allerdings auf eine zweideutige Weise: dadurch daß er sie äußert, auf sie ,anspielt", sie in Gedanken ,durchspielt", bleiben sie neben dem Urteil seiner prinzipiellen Ablehnung terroristischer Gewalt im Text noch gegenwärtig (37). Dem Mescalero gelingt keine rationale Verarbeitung seiner Haßgefühle (38).

Deutlicher ist dieses Problem in der bereits zitierten (Abschieds-)Nr. 179 des Info angesprochen:

„Jeder kennt Todeswünsche - gegen seine Eltern, in Beziehungen, gegen Unterdrükker - das ist nichts ungewöhnliches. Das Unbewußte enthält eine Vielzahl destruktiver Tendenzen, die selbstverständlich gesellschaftlich bedingt sind. Dennoch sollten wir nicht hinter einen bestimmten Standart der Humanisierung und Triebbeherrschung zuriickfallen, zumal uns eine wahrhaft befreite humane Welt vorschwebt."

37 s. P. Brückner, ,Die Mescalero-Affäre“; wo er den Gedankengang des Buback-Nachrufs wie folgt rekonstruiert: ,die Überführung von Gewalt-Phantasien und z.T. chaotischen Einfällen in ein Urteil; die Kritik an Terror und Attentat auf der Basis einer - eingestandenen, daher für viele ,skandalösen" - eigenen spontanen Tendenz zur Gewalt", (S. 30 f.)

38 Insofern reicht die Kritik des Mescalero,,Wir alle müssen davon runterkommen, die Unterdrücker des Volkes stellvertretend für das Volk zu hassen ..." nicht weit genug: Haß ist nicht nur dann illegitim, wenn er in stellvertretender Form auftritt; die richtige politische Kritik an avantgardistischen Konzeptionen gesellschaftlicher Veränderung kann die notwendige ethische Kritik an einer politischen Haltung, die Haß (und sei er ,Klassenhaß") als legitim erscheinen läßt, nicht ersetzen. Eine befreite Gesellschaft kann nicht nur nicht mit der Knarre herbeigeschossen werden, sie kann auch nicht herbeigehaßt werden. Nicht allein die Knarre ist das Problem, sondern die Gedanken dessen, der den Feind be reits im Kopf liquidiert hat, wenn er ihn über Kimme und Korn anvisiert. (Dem kann man auch nicht durch den Verweis auf legitime - letztlich immer Selbstvertejdigungs- - Gewalt in Argentinien ausweichen: Haß kann nie die Grundlage für solche Legitimität sein! 
Die in den letzten drei Abschnitten (VII - IX) anskizzierten Gefahren, die in bestimmten Verhaltensmustern von Teilen der , linken scene" angelegt sind (39), sind natürlich sehr verschiedener Natur und auch von unterschiedlicher (politischer, ethischer) Tragweite. Um Mißverständnisse, die aus der Form (oder Reihenfolge) ihrer Darstellung erwachsen könnten, vorzubeugen: Es besteht $\mathrm{zwischen}$ ihnen auch kein notwendiger Zusammenhang, keinerlei logische Zwangsläufigkeit. Ebensowenig, wie z.B. ohne Zusatzprämissen aus einer radikalen Gesellschaftskritik die Annahme der Legitimität revolutionärer Gewalt oder gar die Forderung nach stellvertretender (terroristischer) Gewalt gegen Repräsentanten ,des Systems" folgt (40), folgt aus den alternativen Lebens-, Verhaltens-, Sprach- und Lektüregewohnheiten im linken Ghetto eine Fetischisierung von Illegalität oder gar ein ,alternatives Fernsehprogramm: die Fortsetzungsserie RAF gegen die Polizei“" (41)

Eher schon kann es psychologische Umschlagspunkte geben, die jedoch von der Entwicklung des politischen Klimas in der BRD insgesamt abhängig sind: Je mehr die sozialistische Linke im öffentlichen Bewußtsein als „Staatsfeind" angesehen und behandelt wird, umso größer wird die Wahrscheinlichkeit sein, daß sich im heute weitgehend diffusen linken,Wir-Gefühl' auch ein Bild „des" Staates als bloßem „Feind" festsetzt. Je weniger in der bundesrepublikanischen Öffentlichkeit grundsätzliche Kritik an der kapitalistischen Verfaßtheit von Staat und Gesellschaft geäuBert werden kann, umso eher wird sich jeder, der solche Fundamentalkritik übt ob er will oder nicht - als ,illegal“ fühlen müssen. Usw. (42)

Diesen Gefahren kann nun keineswegs einfach durch die Formel der ,Einheit der Linken " begegnet werden, die in politischen Diskussionen der letzten Zeit häufig aufgetreten ist (und zumeist von selbstemannten Avantgarden vorgebracht wird,

39 Fraglos habe ich dabei im Sinne einer Argumentation, die auf Gefahren aufmerksam machen - nicht aber sie beschwören will - Einzelaspekte überspitzt. So haben Genoss(inn)en, mit denen ich eine erste Fassung dieses Papiers diskutiert habe, kritisiert, dał ich fast nur die negativen Seiten der ,scene" benenne. Vielleicht. Ich tue dies aber gerade deshalb, weil ich davon ausgehe, daß in den vielfältigen - hier unter dem Sammelwort ,scene" zusammengefaßten - Versuchen alternativer Verkehrs- und Lebensformen Elemente vorhanden sind, die notwendiger Bestandteil sozialistischer Politik sein müssen, gerade weil sie die Trennung von „Bewegung" und „Endziel“ am eigenen Leibe nicht mehr mitmachen und die Vorstellung befreiter, humaner Verhältnisse nicht auf den Tag nach der ,Revolution" verschieben. Gerade jene in der Tat alternativen Formen werden aber durch die Tendenzen zur Selbstisolation, durch bloßes Ghettobewußtsein usw., letztlich wieder zerstört.

40 s. den Leserbrief Ernst Tugendhats „Ist Terrorismus die Konsequenz von Kritik?“, in der Zeit, 23.9.77

41 s. Fichter/Lönnendoncker, a.a.O., wo einem solchen Ineinanderschieben von ,scene-Verhalten" und ,,alternativem Fernsehprogramm" zumindest Vorschub geleistet wird.

42 Inwieweit die Form der aktuell von Teilen der etablierten Machtelite vorangetriebenen Terrorismusbekämpfung ,nicht nur historisch erkämpfte bürgerliche Rechte bedroht, sondern paradoxerweise auch noch mit einiger Sicherheit dazu beiträgt, dem Terror neue Nahrung zu verschaffen", hat Gerhard Vinnai in seinen Überlegungen ,Zur Sozialpsychologie des Terrorismus in der BRD" gezeigt; in: SHL-Info, Nr. 10, S. 38 ff. 
die vorgeben, für „die Linke“" zu sprechen). Denn „die Linke“ als strukturierte pol1tisch-kulturelle Strömung gibt es in der BRD derzeit nicht; und die sehr verschiedenen Strömungen, Traditionslinien und Milieus, die man als , links' bezeichnen kann, lassen sich in ihren Denk-, Handlungs- und Reaktionsmustern keineswegs addieren (43). Auch ist eine ,Vereinheitlichung ${ }^{6}$ der verschiedenen Erfahrungsprozesse (z.B. von Bürgerinitiativen, Gewerkschaftern, studentischen Initiativen, Frauengruppen...), der Handlungsfelder und Aktionsformen, in denen radikale Opposition und anti-kapitalistisches Bewußtsein entstehen kann, für absehbare Zukunft wenig wahrscheinlich.

Darüber hinaus zeigen schon die Argumentationen, mit denen die Forderung nach einer undifferenzierten „Einheit der Linken“ vertreten wird - und in denen meist nach dem empirisch falschen wie politisch verhängnisvollen Schema ,alles links von der Sozialdemokratie" verfahren wird - daß wir unter diesem Motto keineswegs aus den oben skizzierten Gefahren einer ,scene“-Mentalität herauskommen. Gegenüber den autoritären Entwicklungstendenzen in Staat und Gesellschaft müsse sich , die Linke“ - so heißt es - „zusammenschließen“ . . ., um dann irgendwann mal wieder in die Offensive zu kommen. „Dieses „Zusammenschließen“ wird dann oft - und hier treffen wir wieder auf Lagermentalitäten - im Sinne von „die Reihen schließen" verstanden: Politische Kritik an undifferenzierten Vorstellungen von Gewalt im Klassenkampf, klare und harte Distanzierung vom Terrorismus müsse zugunsten jener Einheit zurïckgestellt werden; wer solche Kritik übe, betreibe (bewußt oder unbewußt) das Geschäft der obrigkeitsstaatlichen Illegalisierung anti-kapitalistischer Kräfte innerhalb „der Linken“ weiter ...

Die Einheit, die sich auf diese Weise herstellte, käme jedoch wieder nur zustande durch den ,gemeinsamen Feind", wäre Einheit derjenigen, die ,dagegen" sind. Es würden also allenfalls verschiedene Ghettos (ML-scene, Sponti-scene . . .) unter einem kleinsten gemeinsamen Nenner - unter einem gemeinsamen Transparent oder Flugblatt - addiert, der wieder nur ein negativer wäre, ohne das Problem der eigenen Ghetto-Existenz in neuer Weise anzugehen. Eine solche Einheit untereinander, die selbst nur instrumentellen Charakter hat (alleine sind wir zu wenige), kann dann mit den vielzitierten fortschrittlichen oder liberalen Kräften auch nur noch instrumentell umgehen (Motto: ,Wie kriegen wir zur Verteidigung unserer Freiräume blöde Liberale, die noch an Demokratie glauben?"). Daß einem solchen Kartell der Unzufriedenen gegenüber von seiten der noch bestehenden Reste liberaler und demokratischer Öffentlichkeit in der Tat berechtigtes Mißtrauen besteht, braucht wohl nicht mehr hervorgehoben zu werden.

Worum es bei der Inangriffnahme einer ,politischen Kultur" von Sozialisten also geht, ist nicht allein, daß untẹr den aktuellen politischen Entwicklungstendenzen die Frage nach dem Zusammenhang ,innerlinker" Glaubwürdigkeit mit den Resten (oder Ansätzen) gesamtgesellschaftlicher Kommunikation neu gestellt werden muß. Das Problem ist allgemeiner: Eine ,Bündnispolitik', die nicht immer auch auf

43 vgl. das Nachwort zu Blanke/Christoph/Kreuzberger/Müller/Schäfer, „Über politisches Handeln ...", a.a.O., S. 62 ff, dessen Ausführungen ich mich voll anschließen kann und die ich daher auch nicht zu wiederhoien brauche. 
kommunikativem Handeln beruht, kann gar nicht verstanden werden!

Dies setzt aber eine Identität von Sozialisten voraus, die nicht allein auf einer ,objektiven“ Analyse der Funktionszusammenhänge kapitalistischer Gesellschaften und ihrer Entwicklungstendenzen, nicht allein auf einer ,materialistischen ${ }^{6}$ Prognose der möglichen sozialen Träger (z. B. Proletariat, Jugend . . .) von Fundamentalopposition zur kapitalistischen Gesellschafts- und Staatsordnung gegründet ist, sondern auch auf einer Praxis der Verständigung über alternative Ziele (und die ihnen adäquaten Wege oder Mittel zu ihrer Verwirklichung). Eine solche Praxis der Verständigung aber wird durch den diffusen Solidarisierungszwang ,der Linken“" eher verhindert als befördert.

Was solche „polititsche Kultur" sicher impliziert, ist die Anerkennung der Pluralität der Erfahrungshorizonte und Ansätze, in denen heute gegen ökonomische Ausbeutung und politische Unterdrückung Widerstand geleistet werden kann. Auch die so entstehenden alternativen Kulturen müssen aber von vornherein - wie dies z. B. die Frauenbewegung tut - auf gesellschaftliche Kommunikation, auf Verständlihkeit und Legitimationsfähigkeit ,nach außen' angelegt sein. Nur dadurch können neue Lebens- und Politikformen wirken über eine bloße selbstbezogene Vergewisserung ,der Linken"hinaus. Und hier hören die Fragen und Schwierigkeiten nicht auf. sondern fangen erst an. Doch dies wäre Thema für einen neuen Artikel.

(Jan. 78) 